\title{
Blood Myeloid Dendritic Cells and slanDC in Antiretroviral Therapy- Suppressed HIV-Infected Patients
}

\author{
Miriam Lichtner ${ }^{1,2}$, Raffaella Rossi ${ }^{*}, 2$, Serena Vita ${ }^{2}$, Stefano Savinelli $^{2}$, Marco Iannetta ${ }^{3}$, Claudia Mascia ${ }^{2}$, \\ Raffaella Marocco ${ }^{1,2}$, Camilla Ajassa ${ }^{2}$, Fabio Mengoni ${ }^{2}$, Laura Scorzolini ${ }^{2}$, Claudio Maria Mastroianni ${ }^{1,2}$ \\ and Vincenzo Vullo ${ }^{2}$
}

\author{
${ }^{1}$ Infectious Diseases Unit, Sapienza University, S. M. Goretti Hospital, Latina, Italy; ${ }^{2}$ Department of \\ Public Health and Infectious Diseases, Istituto Pasteur-Fondazione Cenci Bolognetti, Sapienza \\ University, Rome, Italy; ${ }^{3}$ Département Infection, Immunité, Inflammation, Institut Cochin, INSERM \\ u1016, Paris, France
}

\begin{abstract}
Myeloid dendritic cells (mDCs) play a complex role in HIV infection regardless of viral replication. The aim of our study was to analyse mDCs in long term antiretroviral therapy (ART)suppressed HIV-infected patients. We evaluated the numbers of mDCs and slanDC in the context of different degree of $\mathrm{CD}^{+} \mathrm{T}$ cell recovery, persistent $\mathrm{T}$ cell activation (as $\mathrm{HLA}-\mathrm{DR}{ }^{+} / \mathrm{CD} 38^{+}$expression) and monocyte-macrophage activation assessed in terms of circulating levels of both sCD14 and sCD163. We enrolled 72 aviremic patients under effective ART and 34 healthy donors (HD). Patients were divided into two groups on the bases of $\triangle \mathrm{CD} 4$, indicating the difference between the value of $\mathrm{CD} 4$ at the time of sampling and CD4 nadir. Higher levels of $\mathrm{mDCs}$ and slanDC were found in patients with $\Delta \mathrm{CD} 4>200 / \mathrm{mmc}$ in comparison to HD. In those patients also an increased level of sCD14 was found, whereas sCD163 seemed to be at normal levels. An augmentation of activated CD4 T lymphocytes was found, although less pronounced in patients with $\Delta \mathrm{CD} 4<200 / \mathrm{mmc}$. In conclusion, our findings showed an expansion of mDCs with a shift to inflammatory slanDC that could sustain both microbial translocation and HIV latency in CD4 T cells.
\end{abstract}

Keywords: slanDC, Myeloid Dendritic Cells, HIV, sCD163, sCD14, immune activation, ART.

\section{INTRODUCTION}

HIV infection is characterized by an immune activation that results in increased cell turnover, immune system exhaustion and progression to AIDS. The general contribution of HIV replication is uncertain, because there is immune activation found also in patients with undetectable viremia [1] and a lack of immune activation in the presence of high viral load in simian immunodeficiency virus infection [2], suggesting that plasma virus replication is not an absolute requirement for systemic immune activation. Moreover, low levels of immune activation persist overtime also in HIV-infected patients under effective antiretroviral therapy (ART) despite CD4 $\mathrm{T}$ cells recovery and viral suppression [3]. Causes and mechanisms of this chronic inflammatory status are poorly understood, but are likely to be multifactorial and encompass innate and adaptive immune responses, with T-cell- and monocyte/macrophageassociated markers as well as inflammatory soluble plasma molecules being predictive of disease progression [4]. In this regard, the analysis of HLA-DR/CD38 expression on T-cells and the measurement of circulating levels of soluble CD14 (sCD14) and $\mathrm{CD} 163$ (sCD163) are increasingly being recognized as important parameters to assess the degree of T-lymphocytes and monocyte/macrophages activation during the course of HIV infection [5,6].

*Address correspondence to this author at the Department of Public Health and Infectious Diseases, Sapienza University of Rome, Italy. Piazzale Aldo Moro 5, 00155, Rome, Italy; Tel. +390649970313; Fax +390649972625;

E-mail: raffaella.rossi@hotmail.it
Persistent $\mathrm{T}$ and myeloid cell activation are present even in patients with successful HIV suppression, and are linked to non-AIDS morbidity and mortality $[7,8]$. In this context the contribution of dendritic cells (DCs) in sustaining HIVassociated immune activation is not completely understood.

DCs participate in the immune dysregulation characteristic of chronic HIV infection, through the secretion of inflammatory cytokines and interferons, and DCs also alter $T$ cell proliferation and differentiation [4]. Myeloid (mDCs) and plasmacytoid dendritic cells (pDCs) decrease rapidly during the first weeks of HIV infection and their circulating levels are only partially restored after the beginning of ART, especially for pDCs $[9,10]$. In addition, a low baseline pDCs count predicts virological failure in ART treated patients, despite CD4+ T cells recovery [11].

A third subset of DCs was identified in peripheral blood using the monoclonal antibody M-DC8, which binds to 6sulfo LacNac (slan), a carbohydrate moiety of the P selectin glycoprotein ligand 1 (PSGL-1) $[12,13]$. SlanDC cells seem to derive from the pro-inflammatory $\mathrm{CD} 14+\mathrm{CD} 16+$ monocytes [14] and to contribute to the pathogenesis of chronic inflammatory diseases such as Crohn's disease, rheumatoid arthritis and psoriasis, since they infiltrate the inflamed ileal mucosa, skin and synovial tissue $[15,16]$.

Recent data suggest a critical role of $\mathrm{mDCs}$ and in particular of slanDC in driving immune activation in viremic HIV-infected patients [17]. On the other hand, the increased microbial translocation with release of LPS and inflammatory cytokines could represent one of the major 
stimuli for $\mathrm{mDC}$ generation and activation during HIV infection. The high degree of plasticity associated with the high capacity of recruitment at tissue-level, frame the mDCs and slanDC as potential key effector cells involved in inflammatory HIV-mediated disorders.

In the present study, we analysed the $\mathrm{mDC}$ compartment in a cohort of patients who have undetectable HIV viremia following long term treatment with ART. In particular, we evaluated the numbers of $\mathrm{mDCs}$ and slanDC in the context of different degree of CD4+ $\mathrm{T}$ cell recovery, persistent $\mathrm{T}$ cell activation (percentage of HLA-DR+/CD38+ double positive $\mathrm{T}$ Lymphocytes) and monocyte-macrophage activation assessed in terms of circulating levels of both SCD14 and sCD163.

\section{METHODS}

\subsection{Study Population}

The study was approved by the local ethics committee and all the recruited subjects provided a written informed consent to participate.

The study population was selected from all patients who presented consecutively to the Outpatients Clinic of the Department of Public Health and Infectious Diseases, Sapienza University of Rome. Inclusion criteria were patients (i) with at least 2 undetectable $(<37$ copies $/ \mathrm{ml})$ determinations of HIV-RNA levels in the previous 12 months, (ii) on a stable ART for at least 2 years and (iii) free from any acute infections at the time of blood collection. Exclusion criteria were pregnancy, age $<18$ years, hepatitis B and $\mathrm{C}$ infection. Based on the selected criteria $72 \mathrm{HIV}$ infected subjects were enrolled in the study. Moreover a group of 37 healthy donors (HD) was included in the study as controls. Demographic, clinical and laboratory data as well as therapies were collected for all participants.

The CD4+ T lymphocytes count at the time of sampling and the CD4 nadir were used to calculate $\triangle \mathrm{CD} 4$, as the difference between the two parameters. According to $\triangle \mathrm{CD} 4$ value, patients were divided into two groups: the first with 56 subjects with $\Delta \mathrm{CD} 4>200$ cells $/ \mathrm{mmc}$ and the second one with 16 subjects with $\Delta \mathrm{CD} 4<200$ cells $/ \mathrm{mmc}$. The value of 200 cells/mmc, as a cut-off for $\Delta \mathrm{CD} 4$, was chosen on the basis of studies that have shown a correlation between this parameter and the risk of clinical progression of the disease $[18,19]$.

\subsection{Enumeration of DCs}

To identify the number of DCs subsets was used a whole blood assay with a protocol no lyse no wash. This method allowed us to quantify the absolute number of cell subsets using the MACSQuant Analyser. $50 \mu 1$ of whole blood were stained with a mixture of antibodies following titration: CD3-PerCP, CD20-PerCP, CD14-PerCP, CD56-PerCP, HLA-DR-APC and M-DC8-FITC (all from Miltenyi Biotec, Germany), CD235a-PerCP and CD123-BV (BioLegend, Inc, USA), CD11c-PE (BD Biosciences Pharmagen, Italy). After 30 minute incubation, at room temperature, in the dark, the lyse solution was added and, after an additional 10 minute incubation, data were acquired using the flow cytometer.
Before data acquisition began a calibration was performed, using MACSQuantTM Calibration Beads (Miltenyi Biotec, Germany). Cells were run on a MACS-Quant Analyser and analysis was performed using MACSQuantifyTM software (Miltenyi Biotec, Germany). Peripheral blood mononuclear cells (PBMCs) (R1 gate) were gated in a dot plot of Side Scatter Channel (SSC) versus Forward Scatter Channel (FSC). CD3, CD20, CD14, CD56 and CD235a (lineage) surface markers were used to exclude $\mathrm{T}$ and $\mathrm{B}$ lymphocytes, monocytes, NK cells and erythrocytes, respectively. From R1 gate, total DCs were defined as HLA-DR+ Lineage- (R2 gate). On R2 gate, dendritic cell markers CD123 and CD11c were used to define pDCs (R3 gate) and $\mathrm{mDC}$ (R4 gate) respectively. On mDCs gate we identified slanDC as cells $\mathrm{M}-\mathrm{DC} 8+$ and $\mathrm{CD} 11 \mathrm{c}+$ positive.

\subsection{CD4+ and CD8+ Activation}

Activation markers of CD4+ and CD8+ T lymphocytes were evaluated by staining $50 \mu \mathrm{l}$ of whole blood with a mix of pre-titred monoclonal antibodies containing CD45VioBlue, HLA-DR-FITC, CD38-APC, CD8-PerCP and CD4-PE (all from Miltenyi Biotec, Germany). After 20 minute incubation, at room temperature and in the dark, lyse solution was added and after additional 10 minute incubation, data were acquired using MACS-Quant Analyser and then analysed by MACSQuantifyTM software (Miltenyi Biotec, Germany). SSC and CD45 were used to identify lymphocytes. In lymphocytes, CD4 and CD8 were gated. Finally, we gated HLA-DR+/CD38+ double positive CD4+ and $\mathrm{CD} 8+\mathrm{T}$ cells to identify activated lymphocytes.

\section{4. sCD14 and sCD163 Plasma Levels}

Plasma samples were frozen at $-80^{\circ}$ until analysed. Soluble immune activation markers sCD14 and sCD163 were measured by ELISA (Quantikine ${ }^{\circledR}$ ELISA, R\&D Systems, Inc. Minneapolis, USA), according to manufacturer's instructions. All samples were tested in double.

\subsection{Statistics}

For statistical analysis, SPSS version 20.0 for windows (SPSS Inc., Apache Software Foundation, Chicago, Illinois) was used. Statistical comparison between groups was done using the Kruskal-Wallis test followed by Dunnett's post hoc test to detect differences between all groups. Spearman's rank correlation coefficient was calculated to determine associations between variables. $\mathrm{P}$ value $<0.05$ was considered statistically significant.

\section{RESULTS}

\subsection{Demographical, Clinical and Laboratory Findings}

72 HIV infected subjects (40 males and 32 females) were enrolled into the study, with the following characteristics (data are expressed as median and ranges): age 51 years (2379), CD4 nadir 107/mmc (4-613), actual CD4 550/mmc (921550), estimated years to be living with HIV infection 14 (226). Regarding ART, 26 patients were under a triple combination therapy containing a protease inhibitor, 34 
containing a non nucleoside reverse transcriptase inhibitor and 12 containing an integrase inhibitor. The median (range) period of continuous HIV viral suppression was 3 years (1$7)$. The HD were 37, 18 males and 19 females, with a median age of $42(20-54)$.

\subsection{Enumeration of $\mathrm{mDC}$ and slanDC}

In all HIV-infected patients, $\mathrm{mDC}$ levels were not different compared to HD (cells $/ \mathrm{ml}$ median, range, 27000/ml, 531-92135 vs 20008/ml, 7760-57900 p=0.09).

Stratifying subjects according to $\triangle \mathrm{CD} 4$, we found that the absolute number of mDCs was significantly lower in patients with $\Delta \mathrm{CD} 4<200$ cells $/ \mathrm{mmc}$, compared to patients with $\Delta$ CD4 $>200$ cells $/ \mathrm{mmc} \quad(16750 / \mathrm{ml}, 1657-42787 \quad v s$ $32271 / \mathrm{ml}, 531-92135 ; \mathrm{p}<0.001)$. Interestingly, the group of $\Delta$ CD4 $>200$ cells $/ \mathrm{mmc}$ showed a number of mDCs higher than HD (32271/ml, 531-92135 vs 20008/ml, 7760-57900 $\mathrm{p}=0.01$ ) (Fig. 1A). The median percentage of $\mathrm{mDCs}$, within total PBMCs, was higher in patients with $\Delta C D 4>200$ cells $/ \mathrm{mmc}$ compared to those with $\Delta \mathrm{CD} 4<200$ cells $/ \mathrm{mmc}$ $(0,63 \%$ vs $0.3 \%, \mathrm{p}=0.006)$ and with $\mathrm{HD}(0,63 \%$ vs $0.41 \%$, $\mathrm{p}=0.004)$.

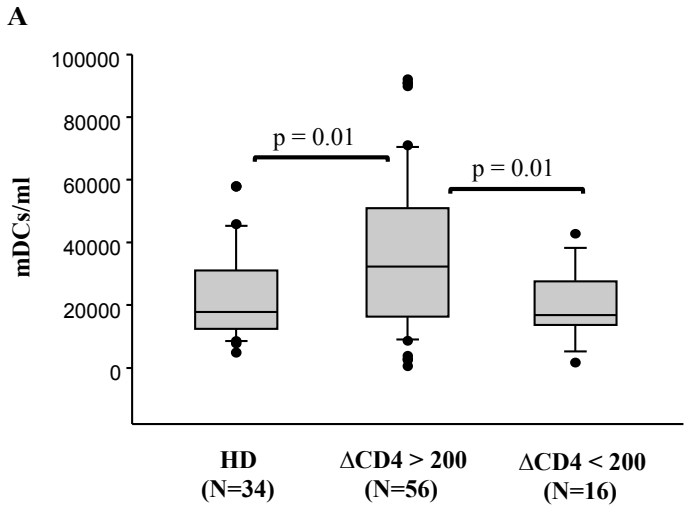

B

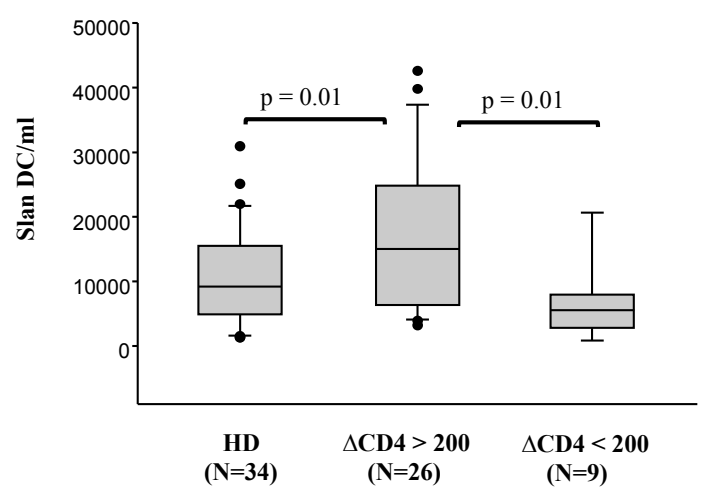

Fig (1). Enumeration of myeloid DC and slanDC in the study population according to $\Delta$ CD4. DCs were measured using a whole blood assay no lyse no wash. Box Plots represent circulating mDCs (A), M-DC8 (B) in patients with $\Delta$ CD4 $>200$ cells $/ \mathrm{mmc}$ and $\Delta \mathrm{CD} 4<200$ cells $/ \mathrm{mmc}$ in comparison to healthy donors. Boxes show 25th, 50th (median) and 75 percentile and wiskers 10 and 90th percentile. Dots represent outlying values. Statistical significance was analyzed by using Kruskal-Wallis test. HD: healthy donors.
In a subgroup of 35 patients we evaluated slanDCs, that were increased in patients with $\Delta C D 4>200$ compare to HD and in patients with $\Delta \mathrm{CD} 4<200(15050 / \mathrm{ml}, 3200-42600 v s$ $8830 / \mathrm{ml}, \quad 1300-30900 \mathrm{p}=0.01$ and $5600 / \mathrm{ml}, 840-20700$ $\mathrm{p}=0.01$ ) (Fig. 1B). The median percentage of slanDCs was higher in patients with $\Delta \mathrm{CD} 4>200$ cells $/ \mathrm{mmc}$ compared to those with $\Delta \mathrm{CD} 4<200$ cells $/ \mathrm{mmc}(0,24 \%$ vs $0.08 \%, \mathrm{p}=0.02)$ and to $\operatorname{HD}(0,24 \%$ vs $0.12 \%, \mathrm{p}=0.01)$.

Moreover, we defined the proportion of $\mathrm{mDC}$ coexpressing M-DC8 markers, computing the percentage of slanDC and M-DC8 negative cells on $\mathrm{mDC}$. We found an increasing trend of slanDCs in patients with $\triangle C D 4>200(49 \%, 9-75)$ in comparison with HD $(39 \%, 5-93)$ and subjects with $\Delta \mathrm{CD} 4<$ 200 (34\%, 8-57) (Fig. 2A).

No differences were observed in pDCs count in patients and HD (6040, 640-20826 vs 6222, 3100-14900, $\mathrm{p}=0.8)$, even stratifying according to $\triangle \mathrm{CD} 4 \quad(\triangle \mathrm{CD} 4<200 \quad v s$ $\Delta \mathrm{CD} 4>200,4735,640-19115$ vs $6579,1120-20826)$ and between $H D$ and $\Delta C D 4<$ and $>200(p>0.05)$. The median percentage of pDCs, did not show differences between groups.

\subsection{CD8+ and CD4+ Immune Activation}

All HIV positive patients showed a significant increase in HLA-DR and CD38 expression on CD4+ cells compared to HD $(1.8 \%$, vs $1.04 \%, \mathrm{p}=0.003)$. The group of patients with $\Delta C D 4<200$ showed the highest level $(2.37 \%$ vs $1.04 \%$, $\mathrm{p}=0.003$ ), although even in patients with $\Delta \mathrm{CD} 4>200$, a higher percentage of CD38 and HLA-DR coexpression compared to HD was observed $(1.76 \%$, vs $1.04 \%, \mathrm{p}=0.01)$ (Fig. 3). There were no significant differences in the percentages of HLA-DR+/CD38+ CD8+ T cells between HIV patients and HD $(0.8 \%$ vs $1.24 \%, \mathrm{p}=0.6)$, neither according to CD4 recovery $(\Delta \mathrm{CD} 4>200$ vs $\Delta \mathrm{CD} 4<200$ : $0.7 \%$, vs $1.67 \%, \mathrm{p}=0.6$ ).

\section{4. sCD14 and sCD163 Plasma Levels}

sCD163 levels were significantly higher in all HIV subjects compared to HD (574 ng/ml, 58-2364 vs $377 \mathrm{ng} / \mathrm{ml}$, 215-1026; $\mathrm{p}=0.03)$, sCD163 was significantly increased in patients with $\triangle \mathrm{CD} 4<200$, compared with subjects with $\Delta \mathrm{CD} 4>200$ and HD (853 ng/ml,150-2310 vs $548 \mathrm{ng} / \mathrm{ml}, 58-$ 2364 and $v s 377 \mathrm{ng} / \mathrm{ml}, 215-1026 ; \mathrm{p}=0.03$ and $\mathrm{p}=0.01$ ) (Fig. 4A).

sCD14 levels were higher in HIV patients compare to HD (1989 ng/ml, 210-4799 vs 855 ng/ml, 290-2467; $\mathrm{p}=0.005$ ), and subjects with $\Delta C D 4>200$ had even a higher level of sCD14 in comparison with patients with $\triangle \mathrm{CD} 4<200$ (2139 ng/ml, 210-4799 vs $970 \mathrm{ng} / \mathrm{ml}, 510-2779, \mathrm{p}=0.04)$ (Fig. 4B).

\subsection{Correlation Analysis}

We observed a positive correlation between the absolute number of $\mathrm{mDCs}$ and slanDC $(\mathrm{r}=0.773, \mathrm{p}<0.001)$, between $\triangle \mathrm{CD} 4$ and slanDCs $(\mathrm{r}=0.413, \mathrm{p}=0.02)$ and $\mathrm{pDCs}(\mathrm{r}=0.379$, $\mathrm{p}=0.001)$ and between HLA-DR+/CD38+ CD4+ and HLA$\mathrm{DR}+/ \mathrm{CD} 38+\mathrm{CD} 8+(\mathrm{r}=0.37, \mathrm{p}=0.006)$. 
$\mathbf{A}$

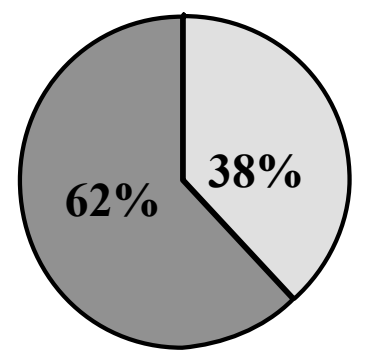

HD

$(\mathrm{N}=34)$
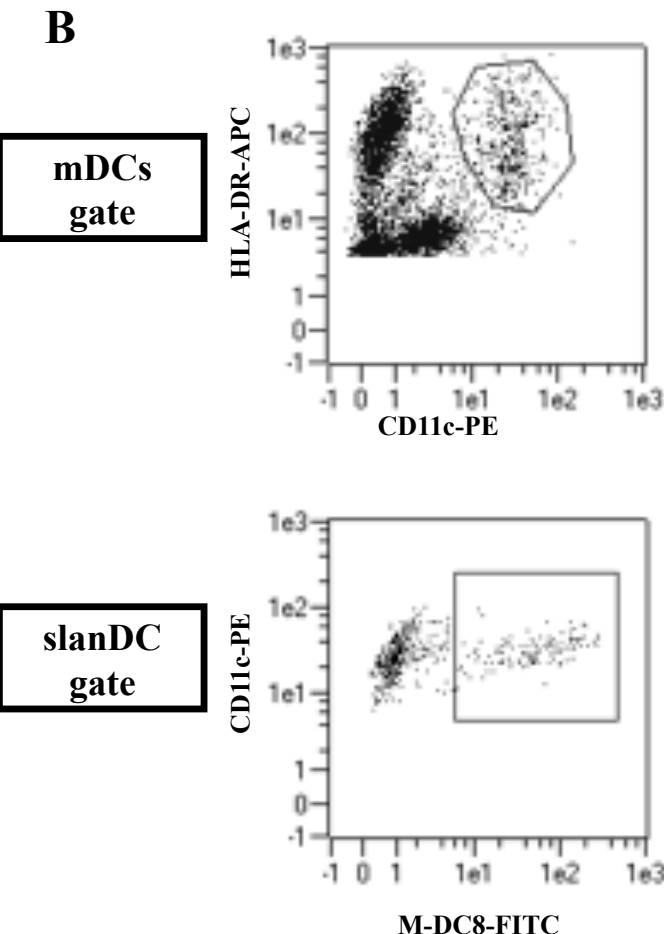

M-DC8-FITC
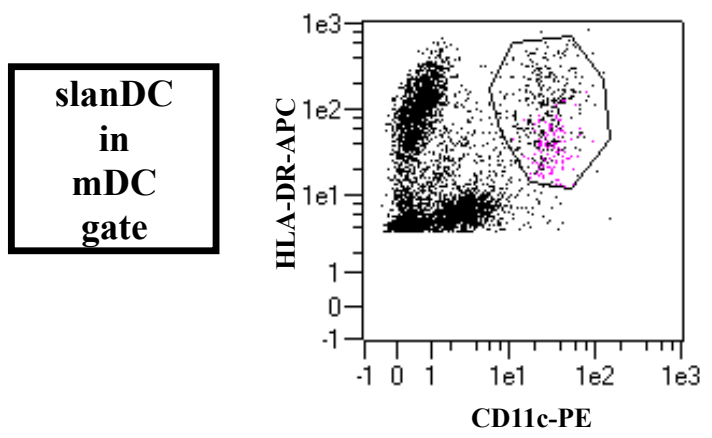

\section{Healthy Donors}

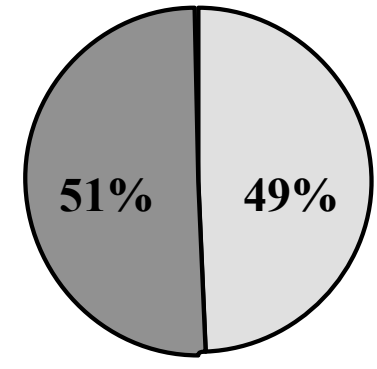

$\Delta$ CD4 $>200$ cells $/ \mathbf{m m c}$

$(\mathrm{N}=26)$
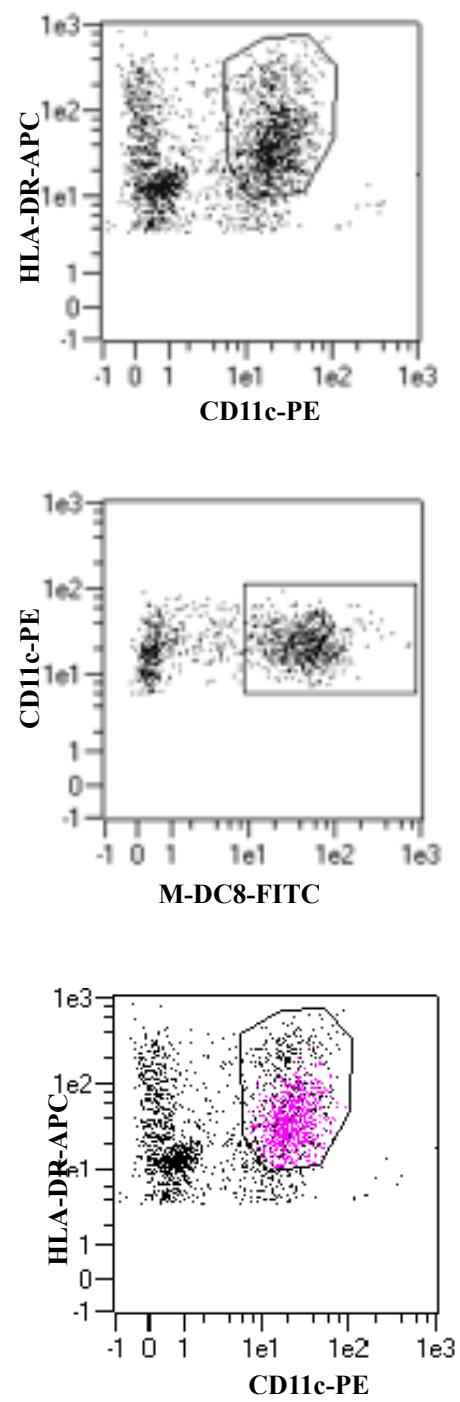

A subject with $\Delta$ CD4 $>200$ cells $/ \mathrm{mmc}$
$M_{-D C 8}^{\text {neg }}$ slanDC

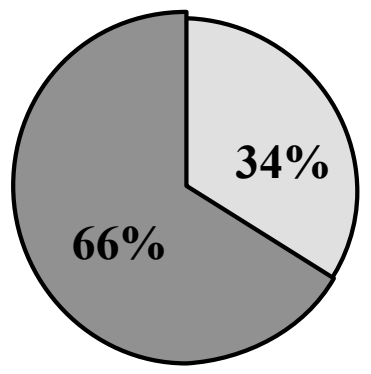
$\triangle$ CD4 $<200$ cells $/ \mathbf{m m c}$ $(\mathrm{N}=9)$
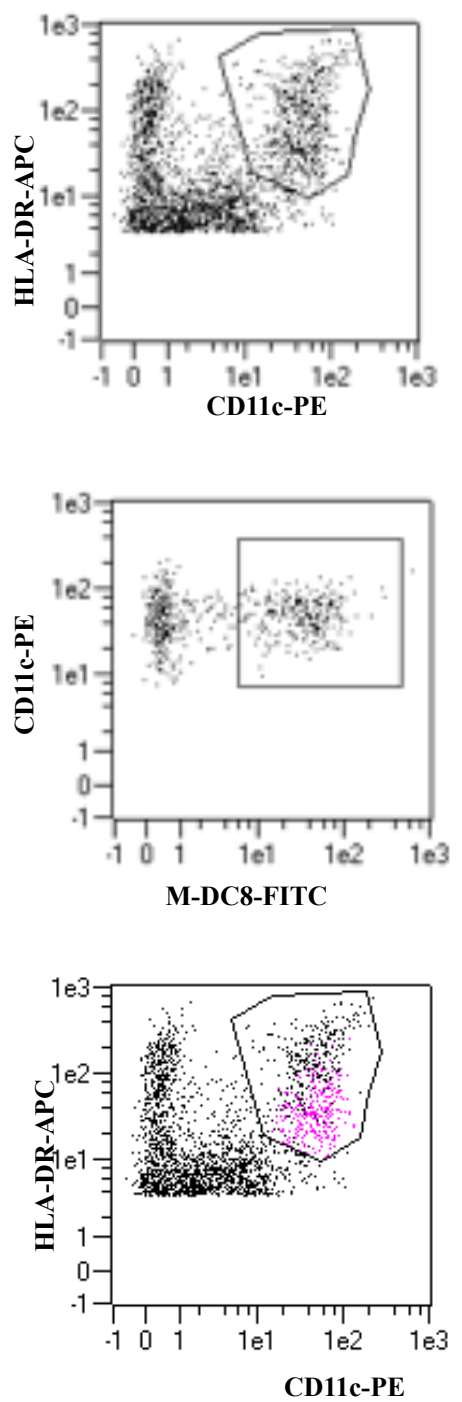

A subject with $\Delta$ CD4 $<200$ cells/mmc

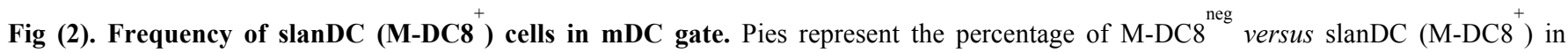
healthy donors and patients with $\Delta \mathrm{CD} 4>200 \mathrm{cells} / \mathrm{mmc}$ and $\Delta \mathrm{CD} 4<200 \mathrm{cells} / \mathrm{mmc}(\mathbf{A})$. Fig. (2B) shows three representative analysis from an healthy donor (left panel), a subject with $\Delta \mathrm{CD} 4>200$ (middle panel) and a subject with $\Delta \mathrm{CD} 4<200$ (right panel). Dot plots identify mDC, slanDC, and slanDCs within mDCs gate in a cytofluorimetric backgating analysis using different colour. 
Regarding sCD163 and $\mathrm{pDCs}$ we found a negative correlation $(\mathrm{r}=-0.241, \mathrm{p}=0.04)$. No other important correlations were found.

Concerning clinical parameters, we found a positive correlation between the value of SCD14 and the years of HIV infection $(\mathrm{r}=0.3, \mathrm{p}=0.01)$ and between $\mathrm{sCD} 163$ and age $(\mathrm{r}=0.305, \mathrm{p}=0.01)$. No other correlations were found (Tables 1 and 2).

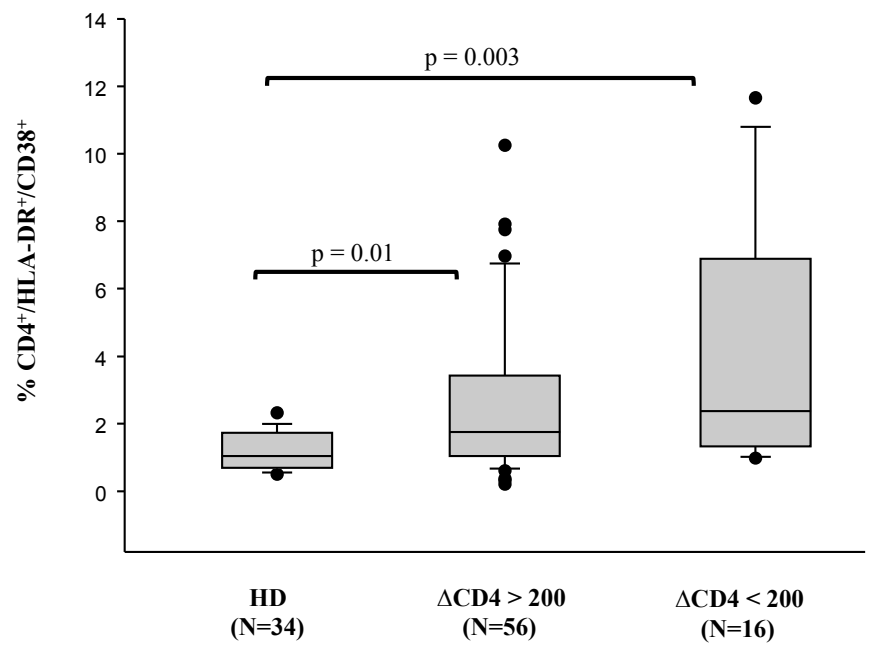

Fig. (3). HLA-DR+/CD38 ${ }^{+} \mathrm{CD}^{+}{ }^{+} \mathrm{T}$ cells according to $\triangle \mathrm{CD} 4$. Box plots represent the percentage of HLA-DR /CD38 CD4 T cells in patients and healthy donors. Activation of CD4 T lymphocytes was analyzed by using a whole blood assay no lyse no wash. Boxes show 25th, 50th (median) and 75 percentile and wiskers 10 and 90th percentile. Dots represent outlying values. Statistical significance was analyzed by using Kruskal-Wallis test. HD: healthy donors.

\section{DISCUSSION}

DCs are the most efficient antigen-presenting cells (APCs), expressing high levels of major histo-compatibility complex (MHC) class II molecules, with the ability to regulate both innate and adaptive immune response towards pathogens [20]. They have the capacity to localize to various tissues/organs, and migrate to lymphoid tissues after antigen acquisition, where they secrete cytokines and initiate immune response [21].

There are two major subsets of DCs, mDCs and pDCs, which differentiate from precursors found in the bone marrow and migrate to the periphery as immature cells. The effects of HIV infection on pDC have been widely investigated and previous data indicate that this DC subset is persistently impaired in number and function despite effective ART. On the other hand, there has been a renewed interest in the $\mathrm{mDC}$ subset that seems to play a complex role in HIV infection. $\mathrm{mDC}$ are involved in multiple processes of the immune response including the stimulation, maintenance and control of both immunity and inflammation. In addition, $\mathrm{mDC}$ cells are able to act as immune sentinels to detect and regulate aberrant immune activation, induce tolerance, avoiding autoimmune diseases [21]. A cytotoxic function was also attributed to $\mathrm{mDC}$ in both neoplastic [22] and other viral infections beyond HIV, such as Ebola and measles $[23,24]$.
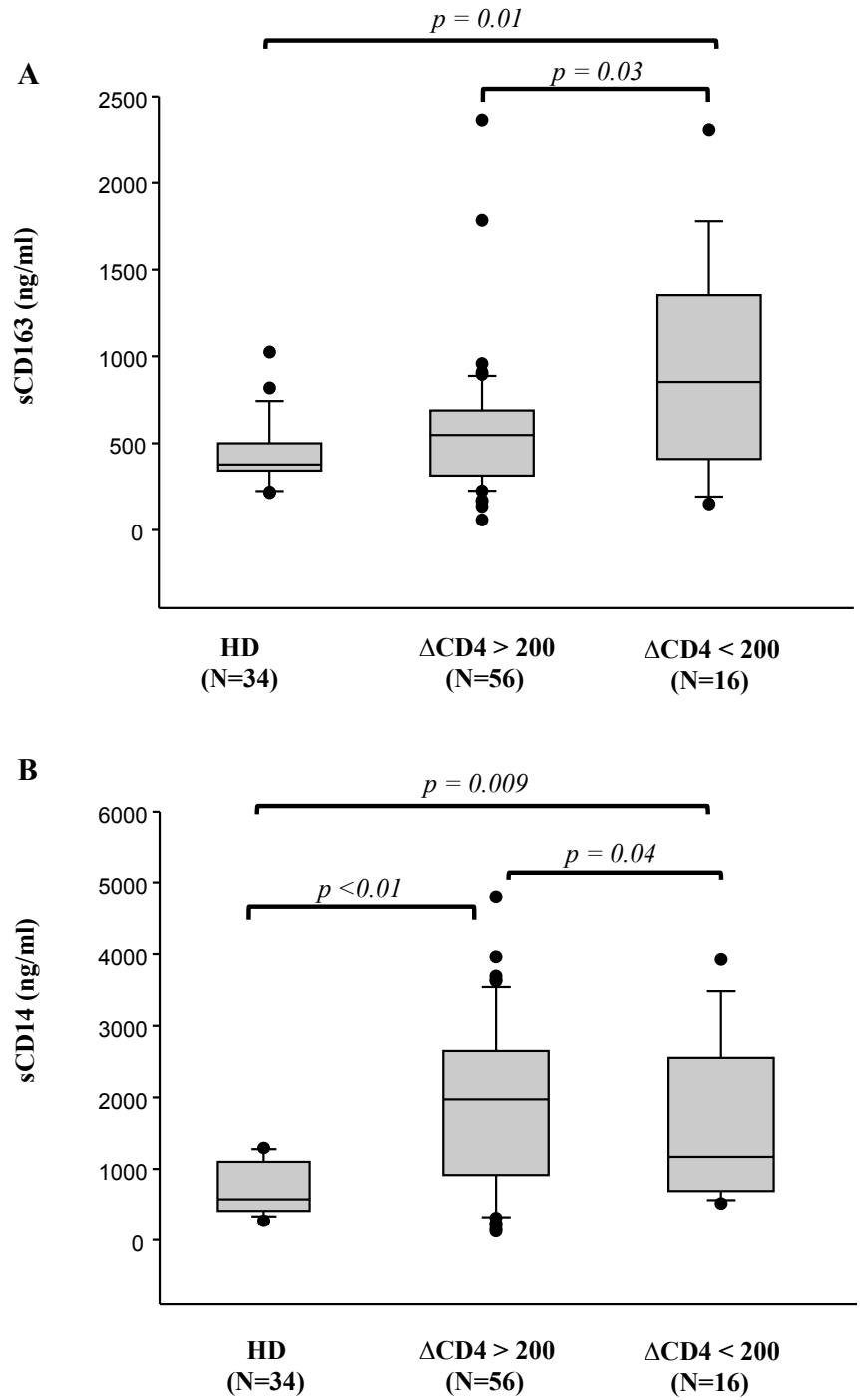

Fig. (4). sCD14 and sCD163 plasma levels. Levels of soluble immune activation markers, sCD14 and sCD163, in plasma were tested by ELISA kit. Dot plots represent the $\mathrm{ng} / \mathrm{ml}$ of soluble markers in patients and control. Lines indicate the median value. Statistical significance was analyzed by using the Kruskal-Wallis test. HD: healthy donors.

In this study we evaluated the role of mDCs and in particular of slanDCs, in a cohort of HIV-infected patients undergoing effective ART. We found that HIV-infected patients with persistent undetectable viremia following longterm ART treatment had increased circulating levels of $\mathrm{mDCs}$ if compared to healthy donors. Interestingly, the most significant increase of these cells was found especially in subjects with a better CD4 recovery $(>200$ cell $/ \mathrm{mmc})$ suggesting a concomitant recovery of both arms of cellular immune system: the innate and the adaptive ones. However, comparing $\mathrm{mDC}$ level of patients with $\Delta \mathrm{CD} 4>200$ to $\mathrm{HD}$ we have observed not just a recovery but a truly mDC expansion. It remains to be clarified the role of this finding in the context of HIV pathogenesis, considering the multiple functions of mDCs. In fact, mDCs are pivotal players in HIV infection, promoting transmission and spread and at the same time are critical for recognizing HIV and initiating immune responses to fight infection [25]. Considering these functional features, it can be assumed that in HIV 
Table 1. Spearman's correlations between subpopulations of dendritic cells, activated Tymphocytes and soluble factors.

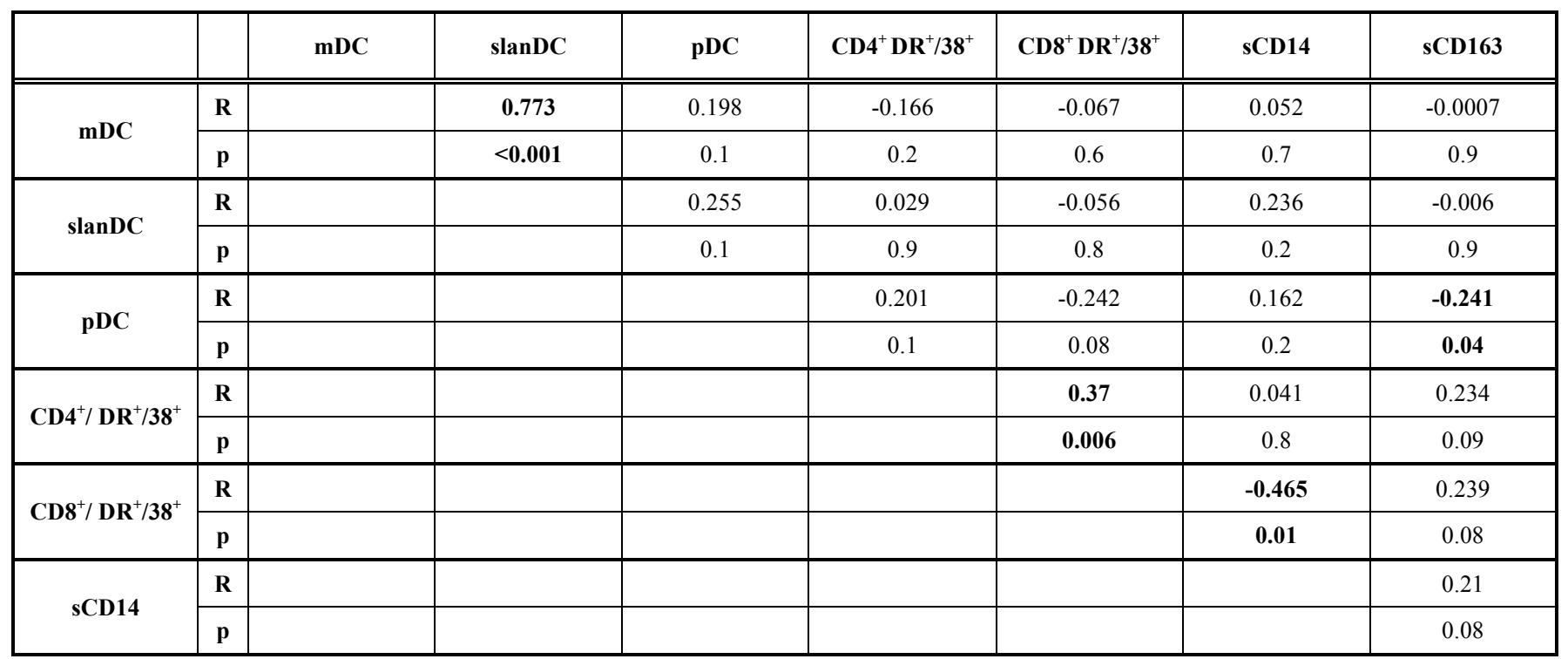

Table 2. Spearman's correlations between subpopulations of dendritic cells, activated Tymphocytes soluble factors and clinical parameters.

\begin{tabular}{|c|c|c|c|c|c|c|c|c|}
\hline & & mDC & slanDC & pDC & $\mathrm{CD4}^{+} \mathrm{DR}^{+} / \mathbf{3 8}^{+}$ & $\mathrm{CD8}^{+} \mathrm{DR}^{+} / \mathbf{3 8}^{+}$ & sCD14 & sCD163 \\
\hline$\Delta \mathrm{CD4}$ & $\mathbf{R}$ & 0.28 & 0.413 & 0.379 & -0.164 & -0.298 & 0.096 & -0.258 \\
\hline \multirow{2}{*}{ Years of HIV } & $\mathbf{R}$ & -0.007 & 0.015 & 0.154 & 0.161 & -0.113 & 0.3 & 0.133 \\
\hline & $\mathbf{p}$ & 0.9 & 0.9 & 0.2 & 0.2 & 0.4 & 0.01 & 0.3 \\
\hline Age & $\mathbf{p}$ & 0.7 & 0.7 & 0.6 & 0.9 & 0.7 & 0.2 & 0.01 \\
\hline \multirow{2}{*}{ CD4 Nadir } & $\mathbf{R}$ & -0.169 & -0.013 & 0.18 & -0.111 & 0.058 & 0.161 & 0.072 \\
\hline & $\mathbf{p}$ & 0.1 & 0.9 & 0.1 & 0.4 & 0.7 & 0.2 & 0.5 \\
\hline
\end{tabular}

pathogenesis, in which the disease progression is driven by high degree of immune activation and inflammation, mDCs may have a significant role [26]. Increase in the number and in the differentiation degree of CD11c+ dendritic cells was observed also in several autoimmune [27] and chronic inflammatory diseases [28,29] suggesting a role of these cells in sustaining inflammation in non microbial diseases. Some authors suggest that mDCs elevation was sustained by microbial translocation or asymptomatic low grade infections that act as a trigger to $\mathrm{mDC}$ differentiation and recruitment [30]. Enumeration of $\mathrm{mDC}$ in blood has been proposed as a predictive tool for monitoring chronic inflammatory disease because the augmentation in blood seems to precede the tissue involvement [31].

Recently, a significant increase in the number of circulating slanDC cells, a major myeloid-derived proinflammatory subpopulation of human blood DCs, has been described in HIV viremic patients compared to both healthy donors and virologically suppressed ART-treated patients [17,32]. Moreover, slanDCs from viremic ART naïve patients are involved in Tumor Necrosis Factor (TNF) $-\alpha$ production in response to LPS, suggesting a pivotal role in the pathogenesis of deleterious immune activation [17]. In a subset of our ART-suppressed HIV-infected patients we also evaluated slanDCs. We observed that the percentage of slanDCs was significantly higher in subjects with $\Delta \mathrm{CD} 4>200$. Conversely, in subjects with $\Delta \mathrm{CD} 4<200$ we found a lower absolute number of $\mathrm{mDCs}$ with a low percentage of slanDC, although the small number of patients in this group could represent a limitation. As expected, slanDCs positively correlated with $\mathrm{mDCs}$ and $\triangle \mathrm{CD} 4$; moreover a positive correlation with pDCs was found. A higher percentage of slanDC, usually known as inflammatory DC, can represent the potential dangerous face of the observed myeloid expansion [16,17,33,34].

In the present study, the analysis of DC subsets was done according to the CD4+ $\mathrm{T}$ cell recovery, but also in the context of persistent $\mathrm{T}$ cell and monocytes/macrophages activation. Concerning $\mathrm{T}$ cells compartment, we observed higher levels of the immune activation markers HLA-DR and $\mathrm{CD} 38$ on $\mathrm{CD} 4+\mathrm{T}$ cells, indicating persist latent HIV infection of these cells. Several studies showed an increased frequency of HLA-DR+/CD38+ CD4+ and/or CD8+ $\mathrm{T}$ lymphocytes in subjects with a persistent viral suppression 
[6,35]. Older age and low CD4 count (and as a consequence lower $\triangle \mathrm{CD} 4$ ) could be the leading factors for immune activation in our cohort of patients. Moreover in an in vitro model of cell to cell contact, resting memory CD4+ T cells seem to be latently infected by HIV only in the presence of mDCs [36]. Thus the high amount of mDCs found in long term ART aviremic patients may facilitate residual ongoing infection of resting T cells and supply the HIV reservoir in the tissues [36]. However, mDCs themselves can serve as reservoir for HIV, in addition to spread the virus [37]. We did not find significant differences in $\mathrm{CD} 8+\mathrm{T}$ lymphocyte activation between HIV infected subjects and uninfected controls, but we observed a direct correlation between the levels of CD4 and CD8 activation. Moreover, the CD8 immune activation was inversely correlated to $\triangle \mathrm{CD} 4$.

In order to assess whether the expansion of $\mathrm{mDC}$ and slanDC was associated to the residual monocyte/macrophage innate immune activation, we measured circulating levels of sCD14 and sCD163. Both sCD14 and sCD163, were found to be increased in patients with both AIDS and non-AIDS related diseases $[7,8,38,39]$. sCD14 exerts an inflammatory effect and is produced mainly by monocytes, whereas sCD163 has an anti-inflammatory modulatory effect and is released by monocytes/macrophages and other tissue cells. A sCD14-dependent pathway has been demonstrated in the LPS activation of DCs, especially with low levels of LPS and has been found in aviremic subjects [40]. In aviremic ART treated subject a persistent low level of LPS was described suggesting that in this population plasma SCD14 is fundamental to maintain residual immune activation [40]. Moreover, a subpopulation of circulating $\mathrm{mDC}$ expresses CD163, together with CD91, and is expanded in HIVinfected patients showing an immunostimulatory role besides its effect in hemoglobin scavenging and anti-inflammation [41].

In our study we found an increase in sCD14 plasma levels, more pronounced in patients with a good CD4 recovery $(\Delta C D 4>200)$ suggesting persistent monocyte activation together with $\mathrm{mDC}$ increase also in aviremic subjects. SCD14 correlated with the years of HIV infection independently from the duration of therapy. The increase of sCD14, in part due to microbial translocation, could act as a stimulus for $\mathrm{mDC}$ and slanDC production and expansion from circulating monocytes. Concomitantly this myeloid expansion could sustain the persistent bowel mucosa damage. In fact slanDCs are found in abundance in inflamed mucosal tissues [33] and they produce large amounts of TNF- $\alpha$, which have an important role in the intestinal epithelial cell destruction, leading to LPS translocation [42] by setting up a vicious circle. Conversely in our study, we did not find a significant correlation between sCD14 level and $\mathrm{mDC}$ or slanDC number, indicating that other factors could be important in the myeloid expansion.

The other soluble factor related to myeloid activation, sCD163, seems to have an important role as a regulator factor with a quenching role in terminating inflammation, especially at tissue macrophage level [43]. In fact, together with its physiological role, which is the inhibition of cellular growth after sequestering extracellular haemoglobin iron [44], sCD163 inhibits the activation and proliferation of $\mathrm{T}$ lymphocytes by limiting the activation of the inflammatory response [43].

In our study sCD163 plasma levels were persistently increased in ART patients with $\triangle C D 4<200$ suggesting a potential immunosuppressive role on $\mathrm{CD} 4+\mathrm{T}$ cells. As already observed $[45,46]$, sCD163 levels correlated with age of patients, suggesting the inability of CD4 recovery in normalizing sCD163 in older HIV subjects. sCD163 levels were not related to $\mathrm{mDC}$ and slanDC cells, but were inversely related to pDCs that in our cohort showed a full recovery.

A limitation of our study is the lack of monocyte analysis, however a recent study showed a persistent alteration of CD16+ monocytes in spite of sCD163 normal levels under ART [8]. Besides we did not evaluate soluble plasma markers strictly related with DCs (such as TNF- $\alpha$, IFN- $\alpha$, IL-12, GM-CSF) since they are produced in a limited amount in plasma from ART treated subjects [17, 47-48]. Ex vivo stimulation with an intracellular assay should be performed to better characterize DCs functionality.

In conclusion HIV-infected people, who have a persistent viral suppression and good CD4 recovery after long term ART, exhibits a large expansion of mDCs associated with a higher proportion of M-DC8 expression, augmentation of sCD14 and CD4 T cells activation despite a normalization of pDC count, CD8 activation and sCD163 levels. The persistence of microbial translocation and immune activation could represent a good environment for the generation of slanDCs and mDCs from monocytes in long term aviremic ART treated patients. On the other hand, this myeloid expansion could sustain both microbial translocation at bowel mucosa level and HIV latency in long lived CD4+ T cells creating a close and vicious circle. A clinical long term follow up of HIV patients with $\mathrm{mDC}$ expansion is needed to understand if this increase is associated with a higher risk of non AIDS events, considering the proinflamatory effect of $\mathrm{mDC}$ at tissue level.

Studies that contribute to an overall understanding of the potentially detrimental roles of $\mathrm{mDCs}$ during viremic or aviremic HIV infection should be strongly encouraged in order to assess the clinical consequence and possible therapeutic approaches.

\section{CONFLICT OF INTEREST}

The authors confirm that this article content has no conflict of interest.

\section{ACKNOWLEDGEMENTS}

This work was supported in part by Gilead Fellowship Program 2011 and SIMIT Grant 2014. The authors thank Prof. Anne Hosmalin, from Immunology Department, Institut Cochin Paris, for the helpful discussion.

\section{REFERENCES}

[1] Hunt PW, Brenchley J, Sinclair E, et al. Relationship between T cell activation and $\mathrm{CD} 4+\mathrm{T}$ cell count in HIV-seropositive individuals with undetectable plasma HIV RNA levels in the absence of therapy. J Infect Dis 2008;197:126-33. 
[2] Silvestri G, Sodora DL, Koup RA, et al. Nonpathogenic SIV infection of sooty mangabeys is characterized by limited bystander immunopathology despite chronic high-level viremia. Immunity 2003;18:441-52.

[3] Vrisekoop N, van Gent R, de Boer AB, et al. Restoration of the CD4 $\mathrm{T}$ cell compartment after long-term highly active antiretroviral therapy without phenotypical signs of accelerated immunological aging. J Immunol 2008;181:1573-81.

[4] Paiardini M, Müller-Trutwin M. HIV-associated chronic immune activation. Immunol Rev 2013;254: 78-101.

[5] Marchetti G, Cozzi-Lepri A, Merlini E, et al. Microbial translocation predicts disease progression of HIV-infected antiretroviral-naive patients with high CD4+ cell count. AIDS 2011;25:1385-94.

[6] Mahalingam M, Peakman M, Davies ET, Pozniak A, McManus TJ, Vergani D. T cell activation and disease severity in HIV infection. Clin Exp Immunol 1993;93: 337-43.

[7] Hattab S, Guihot A, Guiguet M, et al. Comparative impact of antiretroviral drugs on markers of inflammation and immune activation during the first two years of effective therapy for HIV-1 infection: an observational study. BMC Infectious Diseases 2014; $14: 122$.

[8] Castley A, Berry C, French M, Fernandez S, Krueger R, Nolan D. Elevated Plasma Soluble CD14 and Skewed CD16+ Monocyte Distribution Persist despite Normalisation of Soluble CD163 and CXCL10 by Effective HIV Therapy: A Changing Paradigm for Routine HIV Laboratory Monitoring? PLoS One 2014;9:e115226.

[9] Pacanowski J, Kahi S, Baillet M, et al. Reduced blood CD123+ (lymphoid) and $\mathrm{CD} 11 \mathrm{c}+$ (myeloid) dendritic cell numbers in primary HIV-1 infection. Blood 2001;98: 3016-21.

[10] Chehimi J, Campbell DE, Azzoni L, et al. Persistent decreases in blood plasmacytoid dendritic cell number and function despite effective highly active antiretroviral therapy and increased blood myeloid dendritic cells in HIV-infected individuals. J Immunol 2002, 168:4796-801.

[11] Lichtner M, Rossi R, Rizza MC, et al. Plasmacytoid dendritic cells count in antiretroviral-treated patients is predictive of HIV load control independent of CD4+ T-cell count. Curr HIV Res 2008;6:19-27.

[12] Schäkel K, Mayer E, Federle C, Schmitz M, Riethmüller G, Rieber EP. A novel dendritic cell population in human blood: one-step immunomagnetic isolation by a specific $\mathrm{mAb}$ (M-DC8) and in vitro priming of cytotoxic T lymphocytes. Eur J Immunol 1998; 28:4084-93.

[13] Schäkel K, Kannagi R, Kniep B, et al. 6-Sulfo LacNAc, a novel carbohydrate modification of PSGL-1, defines an inflammatory type of human dendritic cells. Immunity 2002;17:289-301.

[14] Siedlar M, Frankenberger M, Ziegler-Heitbrock LH, Belge KU. The M-DC8-positive leukocytes are a subpopulation of the CD14+ CD16+ monocytes. Immunobiology 2000;202:11-7.

[15] de Baey A, Mende I, Baretton G, et al. A subset of human dendritic cells in the $T$ cell area of mucosa-associated lymphoid tissue with a high potential to produce TNF-alpha. J Immunol 2003;170:508994.

[16] Schäkel K, von Kietzell M, Hänsel A, et al. Human 6-sulfo LacNAc-expressing dendritic cells are principal producers of early interleukin-12 and are controlled by erythrocytes. Immunity 2006;24:767-77.

[17] Dutertre CA, Amraoui S, De Rosa A, et al. Pivotal role of MDC8 ${ }^{+}$monocytes from viremic $\mathrm{HIV}$-infected patients in $\mathrm{TNF} \alpha$ overproduction in response to microbial products. Blood 2012;120:2259-68.

[18] Haas DW, Geraghty DE, Andersen J, et al. Immunogenetics of CD4 lymphocyte count recovery during antiretroviral therapy: An AIDS Clinical Trials Group study. J Infect Dis 2006;194:1098-107.

[19] Woelk CH, Beliakova-Bethell N, Goicoechea M, et al. Gene expression before HAART initiation predicts HIV-infected individuals at risk of poor CD4+ T-cell recovery. AIDS 2010; 24:217-22.

[20] Banchereau J, Briere F, Caux C, et al. Immunobiology of dendritic cells. Annu Rev Immunol 2000;18:767-811.

[21] Banchereau J, Steinman RM. Dendritic cells and the control of immunity. Nature 1998;392:245-52.

[22] Lu L, Qian S, Hershberger PA, Rudert WA, Lynch DH, Thomson AW. Fas ligand (CD95L) and B7 expression on dendritic cells provide counter-regulatory signals for $\mathrm{T}$ cell survival and proliferation. J Immunol 1997;158:5676-84.

[23] Vidalain PO, Azocar O, Yagita H, Rabourdin-Combe C, ServetDelprat C. Cytotoxic activity of human dendritic cells is differentially regulated by double-stranded RNA and CD40 ligand. J Immunol 2001;167:3765-72.

[24] Lichtner M, Maranon C, Vidalain PO, et al. HIV type 1-infected dendritic cells induce apoptotic death in infected and uninfected primary CD4 $\mathrm{T}$ lymphocytes. AIDS Res Hum Retroviruses 2004;20:175-82.

[25] Wu L, Kewal Ramani VN. Dendritic-cell interactions with HIV: infection and viral dissemination. Nat Rev Immunol 2006;6:85968.

[26] Wijewardana V, Kristoff J, Xu C, et al. Kinetics of myeloid dendritic cell trafficking and activation: impact on progressive, nonprogressive and controlled SIV infections. PLoS Pathog 2013;9:e1003600.

[27] Gill MA, Blanco P, Arce E, Pascual V, Banchereau J, Palucka, AK. Blood dendritic cells and DC-poietins in systemic lupus erythematosus. Hum Immunol 2002;63:1172-80.

[28] Abla O, Egeler RM, Weitzman S. Langerhans cell histiocytosis: Current concepts and treatments. Cancer Treat Rev 2010;36:354-9.

[29] Chen Y, Tian J, Tian X, et al. Adipose tissue dendritic cells enhances inflammation by prompting the generation of Th17 cells. PLoS One 2014;9:e92450.

[30] Wijewardana V, Soloff AC, Liu X, Brown KN, Barratt-Boyes SM. Early myeloid dendritic cell dysregulation is predictive of disease progression in simian immunodeficiency virus infection. PLoS Pathog 2010; 6:e1001235

[31] Miles B, Abdel-Ghaffar KA, Gamal AY, Baban B, Cutler CW. Blood dendritic cells: "canary in the coal mine" to predict chronic inflammatory disease?. Front Microbiol 2014;5:6.

[32] Almeida M, Cordero M, Almeida J, Orfao A. Different subsets of peripheral blood dendritic cells show distinct phenotypic and functional abnormalities in HIV-1 infection. AIDS 2005;19:261-71.

[33] de Baey A, Mende I, Baretton G, et al. A subset of human dendritic cells in the $\mathrm{T}$ cell area of mucosa-associated lymphoid tissue with a high potential to produce TNF-alpha. J Immunol 2003;170:508994.

[34] Hansel A, Gunther C, Ingwersen J, et al. Human slan (6-sulfo LacNAc) dendritic cells are inflammatory dermal dendritic cells in psoriasis and drive strong TH17/TH1 T-cell responses. J Allergy Clin Immunol 2011;127:787-94.

[35] Peakman M, Mahalingam M, Pozniak A, McManus TJ, Phillips AN, Vergani D. Markers of immune cell activation and disease progression. Cell activation in HIV disease. Adv Exp Med Biol 1995;374:17-26.

[36] Evans VA, Kumar N, Filali A, et al. Myeloid dendritic cells induce HIV-1 latency in non-proliferating CD4+ T cells. PLoS Pathog 2013;9:e1003799.

[37] Lore K, Smed-Sorensen A, Vasudevan J, Mascola JR, Koup RA. Myeloid and plasmacytoid dendritic cells transfer HIV-1 preferentially to antigen-specific CD4+ T cells. J Exp Med 2005;201:2023-33.

[38] Burdo TH, Lentz MR, Autissier P, et al. Soluble CD163 made by monocyte/macrophages is a novel marker of HIV activity in early and chronic infection prior to and after anti-retroviral therapy. $J$ Infect Dis 2011;204:154-63.

[39] Burdo TH, Weiffenbach A, Woods SP, Letendre S, Ellis RJ, Williams KC. Elevated sCD163 in plasma but not cerebrospinal fluid is a marker of neurocognitive impairment in HIV infection. AIDS 2013;27:1387-95.

[40] Verhasselt V, Buelens C, Willems F, De Groote D, HaeffnerCavaillon N, Goldman M. Bacterial lipopolysaccharide stimulates the production of cytokines and the expression of costimulatory molecules by human peripheral blood dendritic cells: evidence for a soluble CD14-dependent pathway. J Immunol1997;158(6):291925.

[41] Maniecki MB1, Møller HJ, Moestrup SK, Møller BK. CD163 positive subsets of blood dendritic cells: the scavenging macrophage receptors CD163 and CD91 are coexpressed on human dendritic cells and monocytes. Immunobiology 2006;211(6-8):40717.

[42] Wang F, Graham WV, Wang Y, Witkowski ED, Schwarz BT, Turner JR. Interferon-gamma and tumor necrosis factor-alpha synergize to induce intestinal epithelial barrier dysfunction by 
upregulating myosin light chain kinase expression. Am J Pathol 2005;166:409-19.

[43] Högger P, Sorg C. Soluble CD163 inhibits phorbol ester-induced lymphocyte proliferation. Biochem Biophys Res Commun 2001;288:841-43.

[44] Weaver LK, Hintz-Goldstein KA, Pioli PA, et al. Pivotal advance: activation of cell surface Toll-like receptors causes shedding of the hemoglobin scavenger receptor CD163. J Leukoc Biol 2006;80:2635.

[45] Hearps AC, Martin GE, Angelovich TA, et al. Aging is associated with chronic innate immune activation and dysregulation of monocyte phenotype and function. Aging Cell 2012;11:867-75.
[46] Martin GE, Gouillou M, Hearps AC, et al. Age-associated changes in monocyte and innate immune activation markers occur more rapidly in HIV infected women. PLoS One 2013;8:e55279.

[47] Malherbe G, Steel HC, Cassol S et al. Circulating biomarkers of immune activation distinguish viral suppression from nonsuppression in HAART-treated patients with advanced HIV-1 subtype C infection. Mediators Inflamm 2014; 2014 :198413.

[48] Vaidya SA, Korner C, Sirignano MN et al. Tumor necrosis factor $\alpha$ is associated with viral control and early disease progression in patients with HIV type 1 infection. $J$ Infect Dis 2014;1;210(7):1042-6. 